\title{
Analysis of Related Factors on the Deformity Correction of Balloon Kyphoplasty
}

\author{
C. Xu, H.-X. Liu, and H.-Z. Xu
}

\begin{abstract}
BACKGROUND AND PURPOSE: Balloon kyphoplasty is a minimally invasive surgical approach for treating vertebral compression fractures, including osteoporotic vertebral compression fractures. The purpose of this study was to determine the effect of balloon inflation and postural reduction on balloon kyphoplasty for the deformity correction of vertebral compression fractures and to explore the correlative factors affecting the deformity correction.
\end{abstract}

MATERIALS AND METHODS: A retrospective study of 72 patients (75 levels) who had undergone balloon kyphoplasty was conducted. Imaging data and clinical features were collected and analyzed. Independent-samples $t$ test analysis was used to find the possible factors affecting deformity correction.

RESULTS: Postural correction in the overextending position significantly increased anterior middle vertebral height, kyphotic angle, and Cobb angle (each $P<.05$ ). There was no significant difference in anterior, middle vertebral height, kyphotic angle, and Cobb angle between postural reduction and balloon dilation (each $P>.05)$. The improvement on the Visual Analog Scale was notable $(P<.05)$. Among a variety of factors, the fracture type (wedge type and biconcave type) affected both the height restoration $(P=.003)$ and kyphotic angle reduction $(P=.007)$. The bone cement injected volume was related to height restoration $(P=.011)$ and kyphotic angle reduction $(P=.018)$.

CONCLUSIONS: Balloon inflation was useless for deformity correction. The deformity correction was attributed to postural reduction with cement strengthening. The most significant factors affecting deformity correction were the fracture type and bone cement injected volume.

ABBREVIATIONS: $\mathrm{VCF}=$ vertebral compression fracture; $\mathrm{KA}=$ kyphotic angle; $\mathrm{BMI}=$ body mass index; $\mathrm{BMD}=$ bone mineral density; $\mathrm{IBT}=$ inflatable bone tamp; $\mathrm{HA}=$ height of anterior vertebra; $\mathrm{HM}=$ height of middle vertebra; $\mathrm{HP}=$ height of posterior vertebra

0 steoporosis is a condition in which the bones lose calcium and become more likely to break. In other words, osteoporosis is a disease of the skeleton in which bones become brittle and prone to fracture. ${ }^{1}$ Bone mineral attenuation reduction is the fundamental basis for the diagnosis of osteoporosis. Osteoporotic vertebral compression fracture (VCF) is the most common complication of osteoporosis. Osteoporosis causes the vertebra to become thin and fragile, increasing the chance of compressing with even minor injury, and, over time, the person with osteoporosis can become hunched over or stooped. During the past 2 decades, balloon kyphoplasty has proved to be a minimally invasive technique for treating VCFs, including osteoporotic VCFs. The inflat-

Received February 26, 2013; accepted after revision April 3

From the Department of Orthopedic Surgery, The Second Affiliated Hospital of Wenzhou Medical College, Wenzhou, Zhejiang, People's Republic of China.

Please address correspondence to Dr Hua-Zi Xu, Department of Orthopedic Surgery, The Second Affiliated Hospital of Wenzhou Medical College, 109 Xueyuanxi Rd, Wenzhou, Zhejiang, People's Republic of China; e-mail: derek1226@163.com

三 Indicates article with supplemental on-line table

http://dx.doi.org/10.3174/ajnr.A3617 able bone tamp (IBT) expands slowly, thus providing a preformed low-pressure cavity for bone cement to be injected in. The aim of this procedure is to relieve pain and to correct deformity of the fractured vertebral body. ${ }^{2-5}$ The benefits of balloon kyphoplasty compared with percutaneous vertebroplasty are better vertebral height restoration and less cement leakage. ${ }^{6}$ Nevertheless, some scholars have observed the existence of dynamic and latent mobility of VCFs. ${ }^{7,8}$ Improvement in fractured vertebral height and restoration of sagittal alignment have been ascribed to postural reduction combined with IBT dilation. However, whether the radiographic changes would be significant after removing the interference of postural factors is unknown.

In this retrospective study, we determined whether IBT inflation offers an additional advantage of realigning the spinal column and regaining height in the index vertebral body and investigated related factors that could contribute to deformity correction.

\section{MATERIALS AND METHODS \\ Patient Data}

We retrospectively reviewed all consecutive patients with VCFs who were treated with balloon kyphoplasty at our institution with 
Table 1: Classifications and data of patients

\begin{tabular}{lc}
\hline \multicolumn{1}{c}{ Classifications } & Data \\
\hline Total No. of cases & $72(75$ fractured vertebrae) \\
Average age, $y$ & $71.47 \pm 1.42(57-86 \mathrm{y})$ \\
$<70$ & $26(36.1 \%)$ \\
$\geq 70$ & $46(63.9 \%)$ \\
Sex & \\
$\quad$ Male & $20(27.8 \%)$ \\
Female & $52(72.2 \%)$ \\
BMD & $19(26.4 \%)$ \\
$>-2.5$ & $53(73.6 \%)$ \\
$\leq-2.5$ & \\
BMI & $30(41.7 \%)$ \\
$<24$ & $42(58.3 \%)$ \\
$\geq 24$ & \\
Type of fracture & $48(66.7 \%)$ \\
Wedge & $24(33.3 \%)$ \\
Biconcave & \\
Cement injected volume & \\
$<6$ mL & $28(38.9 \%)$ \\
$\geq 6$ mL & $44(61.1 \%)$ \\
\hline BMl = weight $(\mathrm{kg}) /$ height $^{2}\left(\mathrm{~m}^{2}\right)$. &
\end{tabular}

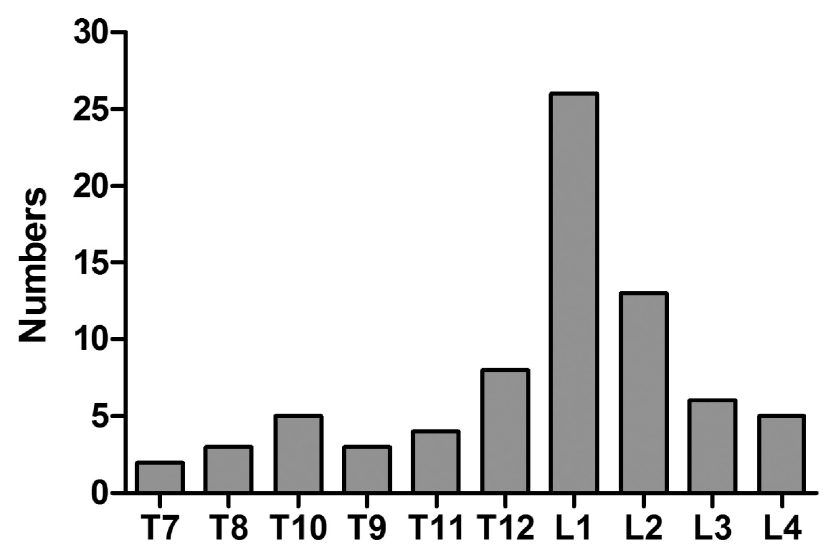

FIG 1. Index vertebra distribution.

verbal informed consent between March 2011 and October 2012. Institutional review board approval and written informed consent were obtained. Selection criteria were 1 ) elderly ( $>50$ years), 2) thoraco-lumbar vertebrae, 3) minor injury or no history of trauma, 4) the constant ache and fatigue in thoraco-lumbar vertebrae that can significantly affect daily life, 5) being diagnosed with osteoporosis or osteopenia by dual-energy x-ray absorptiometry (Lunar Prodigy-DXA; Lunar Radiation, Madison, Wisconsin), 6) imaging (x-ray, CT scan, or MR imaging) showing thoraco-lumbar vertebral morphologic change, and 7) fresh fractures (the duration between presentation or injury and surgery was $\leq 3$ weeks). The exclusion criteria were 1 ) fractures older than 3 weeks' duration, 2) pathologic fracture, 3 ) vertebral body height fully recovered after postural reduction, and 4) MR imaging signal findings only, without the index vertebral morphologic change. Seventy-two consecutive patients were enrolled in the study according to the above criteria. There were $52(72.2 \%)$ women and $20(27.8 \%)$ men, ranging in age between $57-86$ years (mean age, 71.5 years). A total of 75 vertebral bodies were treated in this group (Table 1). The level of the fracture was distributed between T7 and L4, and was most prevalent in the thoraco-lumbar junction (Fig 1). Of all patients treated, 69 had a single vertebral fracture

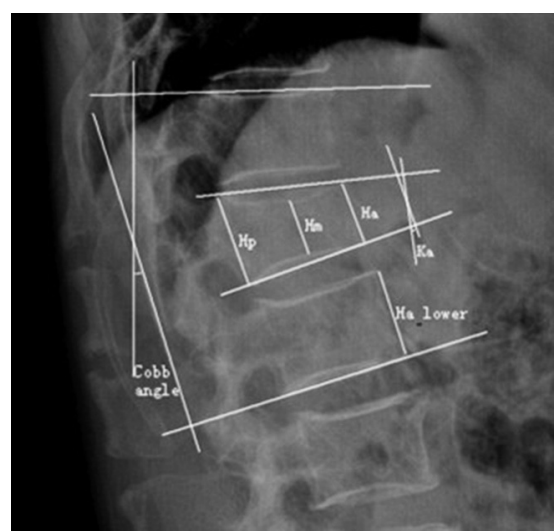

FIG 2. Illustration showing the measurement of anterior (HA), middle $(\mathrm{HM})$, and posterior (HP) portions of fractured vertebral height; kyphotic angle (KA); Cobb angle; and vertebral heights 1 level above (upper) and below (lower) the index vertebra.

and 3 had multiple lesions. The categoric variables, including sex, age, bone mineral density (BMD), body mass index (BMI), fracture type, and bone cement injected volume, were checked and classified (Table 1). Patients were grouped according to categoric variables and the radiographic outcomes for each and were then compared. For all patients, x-ray, CT scan, and MR imaging were performed before surgery and $\mathrm{x}$-ray again after the procedure.

\section{Operative Technique}

The patient was positioned prone on a radiolucent operating bed that allowed for anteroposterior and lateral imaging, with the thoraco-lumbar junction hyperextended by cushions. Cushions were positioned under the iliac crests and both sides of the upper thorax. The operating bed was flexed as needed to increase extension at the thoraco-lumbar junction. General anesthesia was the most comfortable option for patients experiencing pain and was used in all procedures. A $1-2-\mathrm{cm}$ skin incision was made lateral to the desired entry point of the pedicle to percutaneously place an IBT (Shandong Guan-long Medical Utensils Co, Shandong Province, China) into the collapsed vertebral body through a bipedicular approach. IBT inflation was performed to create a low-pressure cavity for cement injection. Inflation continued until the IBT reached 300 psi or the maximum size was reached. The IBTs were removed, and viscous bone cement (polymethylmethacrylate; Tianjin Synthetic Materials Research Institute, Tianjin, China) was injected through the working cannula. The whole therapeutic process was monitored and recorded by the $\mathrm{C}$-arm digitalized $\mathrm{x}$-ray system.

\section{Imaging Parameters and Clinical Outcomes}

Each fractured vertebra was sequentially measured for its height of anterior vertebra (HA), height of middle vertebra (HM), height of posterior vertebra (HP), kyphotic angle (KA), and Cobb angle ${ }^{9}$ in the midsagittal plane on a PACS. Kyphotic angle was the angle defined by the intersection of superior and inferior endplate of the fractured body. Determination of the Cobb angle measurement was taken from the superior endplate of the vertebra 1 level above the treated vertebra to the inferior endplate of the vertebral body 1 level below the treated vertebra. The vertebral heights 1 level above and below the index vertebra were also calculated (measurement method is 
Table 2: Imaging parameters during balloon kyphoplasty $(\bar{x} \pm s)$

\begin{tabular}{lcccc}
\hline & Preoperative & Prone & Balloon Inflated & Postoperative \\
\hline $\mathrm{HA}, \mathrm{mm}$ & $21.30 \pm 5.34^{\mathrm{a}}$ & $26.37 \pm 4.34$ & $26.47 \pm 4.41^{\mathrm{b}}$ & $25.61 \pm 4.50^{\mathrm{c}}$ \\
$\mathrm{HM}, \mathrm{mm}$ & $17.88 \pm 4.65^{\mathrm{a}}$ & $21.57 \pm 4.28$ & $21.76 \pm 4.38^{\mathrm{b}}$ & $21.24 \pm 4.24^{\mathrm{c}}$ \\
$\mathrm{HP}, \mathrm{mm}$ & $27.97 \pm 4.64$ & $27.96 \pm 4.65$ & $28.07 \pm 4.68$ & $27.98 \pm 4.65$ \\
\hline
\end{tabular}

${ }^{a} P<.05$ for preoperative versus prone.

${ }^{b} P>.05$ for balloon inflated versus prone.

$c P<.05$ for postoperative versus preoperative.
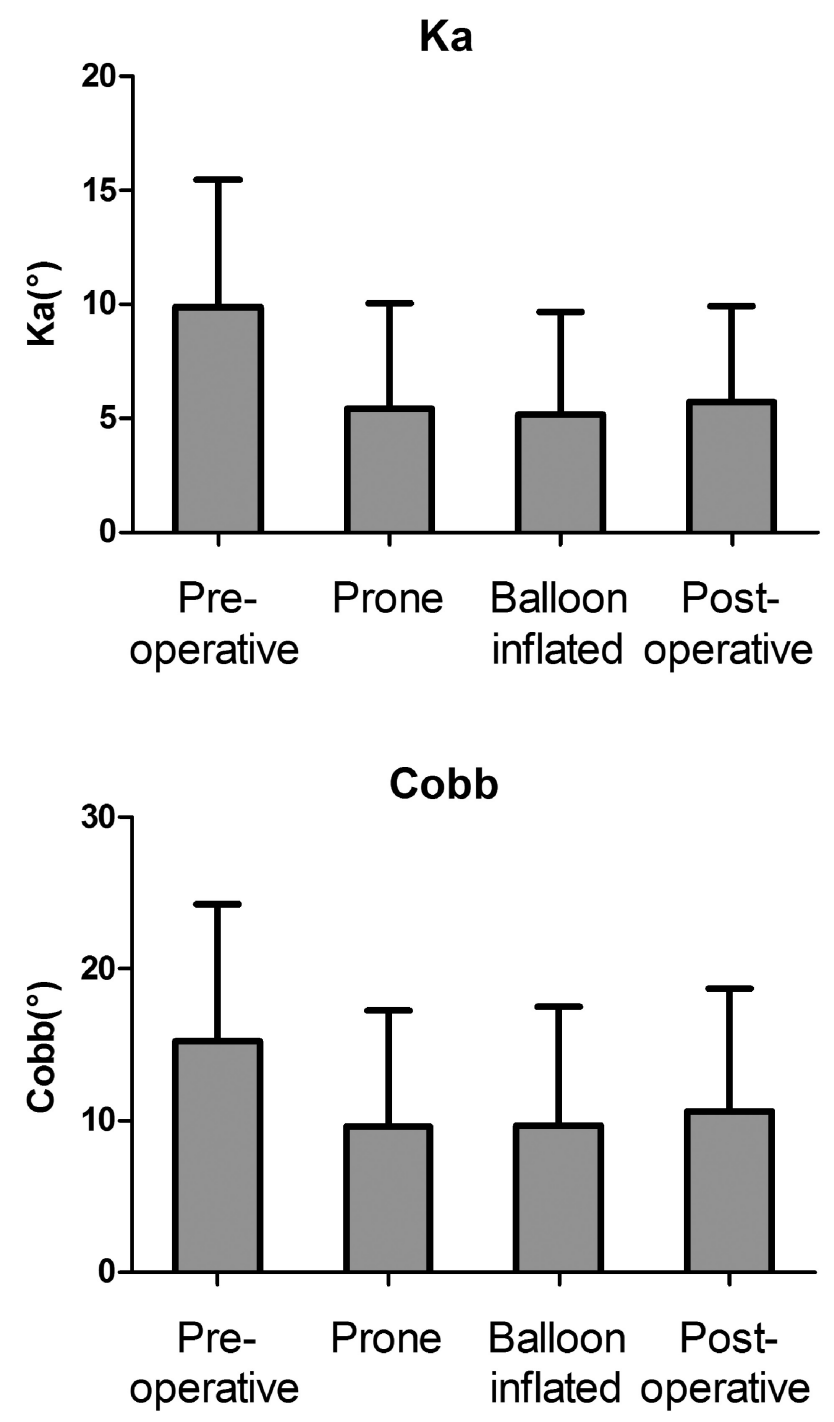

FIG 3. Change of the KA and Cobb angle during balloon kyphoplasty $(-\mathrm{x} \pm \mathrm{s})$. Note: Prone compared with preoperative, $P<$ .05 ; balloon inflated compared with prone, $P>.05$; postoperative compared with preoperative, $P<.05$.

shown in Fig 2). Initial index vertebra height $=\left(\right.$ height $_{\text {upper }}+$ height $\left._{\text {lower }}\right) / 2$. Height restoration ratio was calculated as follows: (height $t_{\text {postop }}-$ height $\left._{\text {preop }}\right) /\left(\right.$ height $_{\text {normal }}-$ height $\left._{\text {preop }}\right) \times 100 \%$. These data were taken from lateral radiographs and analyzed at 4 different time points: 1$)$ preoperative (standing), 2) intraoperatively (placing the patient in prone hyperextension position), 3) after inflation of the IBT, and 4) postoperative (standing). The clinical outcomes were measured with the Visual Analog Scale, which patients were asked to complete before and after surgery. All parameters were measured and recorded by one surgeon.

\section{Statistical Analysis}

All statistical analysis, including mean values and standard deviations, were performed with the use of commercially available software (SPSS 19 for Windows; IBM, Armonk, New York) with the significance level setting at level $P=$ .05. The statistical significance of different time points was assessed with the paired $t$ test. Categoric variables (sex, age, BMD, fracture type, BMI, and bone cement injected volume) were compared and evaluated by means of the independent-sample $t$ test. A value of $P<.05$ was considered to show a statistically significant difference.

\section{RESULTS}

We examined whether patients who were treated with kyphoplasty obtained height restoration and kyphosis rectification. Changes in measured parameters before vertebral augmentation, after postural positioning on the table, after IBT inflation, and after the procedure are presented in Table 2, the On-line Table, and Fig 3. Imaging of a typical case is shown in Fig 4.

Vertebral body height and KA were markedly affected by placing the patient in the hyperextended prone position with extended posture at the commencement of the procedure. Minor further improvement in vertebral height (Table 2, $P>.05$ ), KA (Fig 3, $P>.05$ ), and Cobb angle (Fig 3, $P>.05$ ) were noted after IBT dilation in comparison with the hyperextended position time point.

In a comparison of preoperative and postoperative measurements, significant improvement in compressed vertebral body deformity was noted. HA and HM improved significantly $(P<$ .05 ), from $21.30 \pm 5.34 \mathrm{~mm}$ to $25.61 \pm 4.50 \mathrm{~mm}$, and from $17.88 \pm 4.65 \mathrm{~mm}$ to $21.24 \pm 4.24 \mathrm{~mm}$, respectively. HP was nearly unchanged after treatment, which improved from $27.97 \pm 4.64$ $\mathrm{mm}$ to $27.98 \pm 4.65 \mathrm{~mm}$ with no significance $(P>.05)$. The KA dramatically $(P<.05)$ fell from $9.90 \pm 5.59^{\circ}$ to $5.69 \pm 4.25^{\circ}$. The Cobb angle dropped significantly, from $15.21 \pm 9.05^{\circ}$ to $10.61 \pm$ $8.08^{\circ}(P<.05)$.

Clinical symptom (low back pain), which was assessed by the Visual Analog Scale, significantly $(P=.003)$ decreased from a mean of $8.2 \pm 0.8$ points (range, $7-10$ points) before surgery to $2.1 \pm 1.0$ points (range, $0-4$ points) 1 day after surgery. All patients were satisfied with the results. The mean bone cement injected volume was $6.2 \mathrm{~mL}$ (range, 2.7-8.0 mL). The leakage of cement into paravertebral soft tissue or veins was detected in 6 cases $(8.33 \%)$. However, no complications related to cement leakage were noted.

All patients benefited significantly $(P<.05)$ from being placed in prone position at the time of surgery (Table 2 and Fig 3 ). HA increased from $21.30 \pm 5.34 \mathrm{~mm}$ to $26.37 \pm 4.34 \mathrm{~mm}$ and $\mathrm{HM}$ increased from $17.88 \pm 4.65 \mathrm{~mm}$ to $21.57 \pm 4.28 \mathrm{~mm}$. The KA and Cobb angle separately reduced from $9.90 \pm 5.59^{\circ}$ to $5.41 \pm$ $4.62^{\circ}$ and from $15.21 \pm 9.05^{\circ}$ to $9.61 \pm 7.67^{\circ}$.

The possible correlative factors, including sex, age, BMD, fracture type, BMI, and bone cement injected volume, were classified and analyzed respectively (On-line Table). When comparing the categoric variables, we found that fracture type and bone cement 

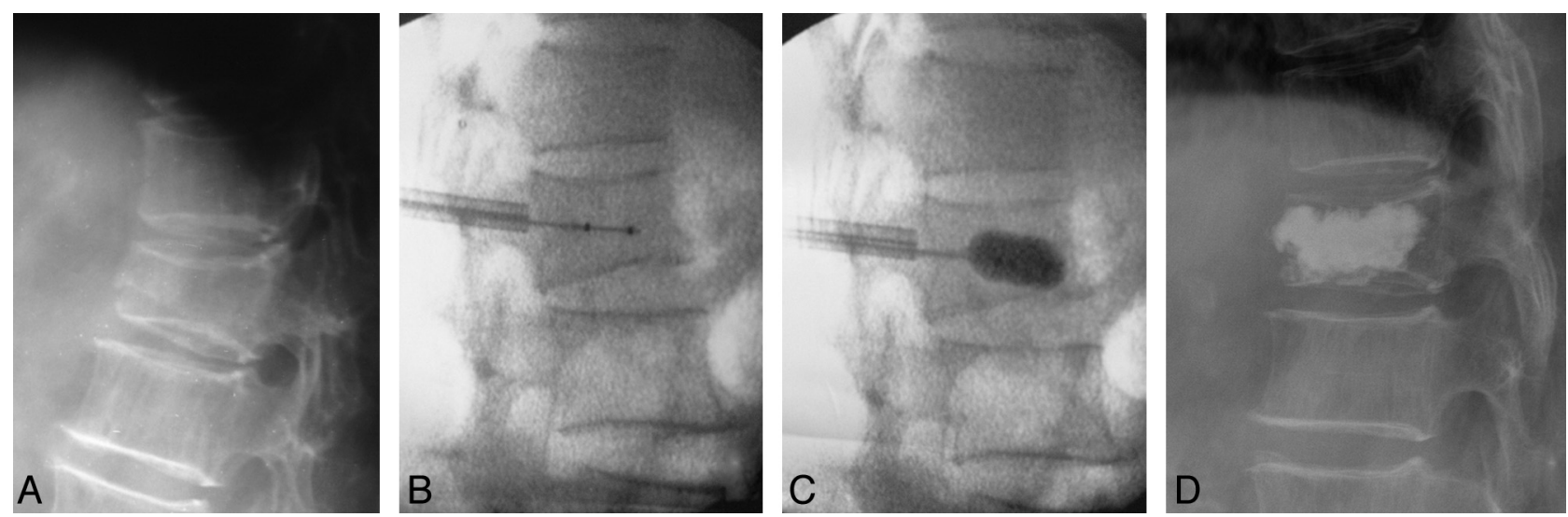

FIG 4. X-ray films of a typical patient: 69-year-old woman with a T12 vertebral compression fracture (wedge type). A, Preoperative standing lateral, $\mathrm{HA}, \mathrm{HM}, \mathrm{KA}$, and Cobb were $20.20 \mathrm{~mm}, 24.57 \mathrm{~mm}, 17.27^{\circ}$, and $14.55^{\circ}$, respectively; $B$, intraoperative prone position lateral, $\mathrm{HA}, \mathrm{HM}, \mathrm{KA}$, and Cobb were $25.33 \mathrm{~mm}, 25.23 \mathrm{~mm}, 8^{\circ}$, and $7^{\circ}$, respectively; $C$, intraoperative IBT inflated, HA, HM, KA, and Cobb were $25.33 \mathrm{~mm}, 25.23$ $\mathrm{mm}, 8^{\circ}$, and $7^{\circ}$, respectively; $D$, postoperative standing lateral, $\mathrm{HA}, \mathrm{HM}, \mathrm{KA}$, and Cobb were $24.82 \mathrm{~mm}, 24.91 \mathrm{~mm}, 8.56^{\circ}$, and $7.12^{\circ}$, respectively.

injected volume showed statistically significant correlation with height restoration $(P=.003, P=.011)$ and KA reduction, $(P=$ $.007, P=.018)$ from the results of independent-samples $t$ test analysis. The kyphosis correction of the wedge type group and $>6$ $\mathrm{mL}$ group were significantly larger than the biconcave type group and the $<6 \mathrm{~mL}$ group, respectively.

\section{DISCUSSION}

The efficacy of balloon kyphoplasty for treatment of VCFs has been widely accepted by most spine surgeons. ${ }^{10,11}$ However, it was recently reported that both percutaneous vertebroplasty and balloon kyphoplasty appeared to be effective at producing rapid relief of pain resulting from VCFs and showed similar orders of magnitude of height restoration. ${ }^{12-15}$ This phenomenon occurred because almost all collapsed vertebral bodies exhibited "dynamic and latent fracture mobility." ${ }^{7,8}$ Nevertheless, some authors ${ }^{16,17}$ support the viewpoint that beyond fracture mobility, a statistically significant further reduction by inflation of the balloon tamp was achieved in every patient.

The results obtained from our study demonstrate that spontaneous reduction in the hyperextended prone position with subsequent cement injection after balloon kyphoplasty resulted in both significant reduction in pain and significant improvement in vertebral deformity, including vertebral height and KA. After hyperextended prone positioning was established, IBT inflation did not appear to significantly further improve vertebral height or KA. Although IBT did not produce additional restoration, it was still an important tool that aided in controlling the distribution of cement during the procedure and compacted the bone fragments, so the bone tunnel was sealed and the incidence of leakage was reduced.

Ligamentotaxis, or manipulative reduction, has been used to prevent the development of postoperative kyphosis in unstable thoraco-lumbar burst fractures. ${ }^{18}$ In our study, we refer to postural reduction as ligamentotaxis, confining patients to the supine position overnight before surgery and placing cushions under the upper chest and the iliac crests during surgery. The anterior column of the compressed vertebra was destroyed, whereas the anterior longitudinal ligament and fibrous ring remained intact, which were important structures in limiting dispersive stress. Through stretching of the anterior longitudinal ligament and the fibrous ring, ligamentotaxis effectively reconstructed the compressed anterior column while adding stability and restoring the sagittal profile. The postural reduction technique created a maximum effect on deformity correction. Research by Cawley et $\mathrm{al}^{19}$ supports our opinion that IBT inflation did not significantly further increase vertebral height or Cobb angle.

To examine the effect of balloon kyphoplasty on the deformity correction for the collapsed vertebral bodies, we collected and analyzed several parameters before and after the operation. According to statistics, the fracture type and cement injected volume might have an effect on the deformity correction, which would help in forecasting the prognosis of the operation.

The selected cases were divided into 2 groups on the basis of the type of fracture-anterior wedge type and biconcave type-by use of a semiquantitative technique developed by Genant et al. ${ }^{20}$ The anterior wedge type fracture was mainly affected by the buckling stress, thus HA and KA appeared obviously deformed. The endplates (upper and lower) of biconcave fractured vertebrae, which are also known as vertical-compression type endplates, were compressed by their adjacent intervertebral disks. Lee et $\mathrm{al}^{21}$ put forward that the compressed vertebral body configuration was only associated with height restoration. However, we found that this factor showed a statistically significant correlation with height restoration and KA reduction. The kyphosis correction of the wedge type group was significantly larger than the biconcave type group. Thus, the aim of treatment for VCFs with small KA was restoring the middle portion height, increasing vertebral strength and stiffness, and quickly relieving pain.

The bone cement injection was terminated when adequate filling of the vertebral body was achieved or if leakage occurred. How do we define "adequate"? The ideal cement injection volume should be required to meet the following 2 conditions: maximum therapeutic effect and minimum side effects. We observed that a larger volume of bone cement achieved better correction effect (On-line Table). Nevertheless, increasing numbers of studies showed $^{22-25}$ that bone cement injection volume had no corre- 
lation with pain relief and had a positive correlation with bone cement leakage rate. In the clinic, many factors influenced the cement injection volume, such as the severity of osteoporosis, the degree of compression, the region of fracture, and so forth. Further study to evaluate these factors would be valuable.

We expected that BMD would have affected the deformity correction and that the change in vertebral height would be especially common in patients with severe osteoporosis because of the presence of unstable nonunion states in these patients, but the result was not as good as we had hoped. This discordance might be limited by the number and age of patients. Surgical treatment for VCFs does not address continued loss of bone attenuation or the progressive increase in fracture risk caused by underlying osteoporosis. Therefore, patients with confirmed osteoporotic VCFs should receive anti-osteoporosis treatment, such as a bisphosphonate, supplemented with adequate doses of calcium and vitamin $\mathrm{D}$, and should pay more attention to the adjacent vertebral fracture after the balloon kyphoplasty. ${ }^{26}$

As short-term observational research from a single center, the lack of a sufficient number of cases and long-term effects assessment are limitations of this study. Although all of the data were measured accurately by one spine surgeon, some measurement errors are still inevitable.

\section{CONCLUSIONS}

Balloon kyphoplasty is a minimally invasive surgical approach for treating VCFs, including osteoporotic VCFs. It can provide an effective and safe therapeutic effect for VCFs with low BMD. Deformity correction of the index vertebral body in early fractures appears to occur as the result of hyperextended postural reduction with subsequent cement strengthening. IBT does not appear to contribute significantly to additional deformity correction in these early fractures when maximum hyperextension postural correction is used. In view of these facts, how to make the selection depends on cost and the spine surgeon's skill. The predominant correlative factors affecting deformity correction were the fracture type and cement injected volume.

\section{ACKNOWLEDGMENTS}

We thank Mr Shi at the Second Affiliated Hospital of Wenzhou Medical College for support and technical assistance.

\section{REFERENCES}

1. Lindsay R, Burge R, Strauss DM. One year outcomes and costs following a vertebral fracture. Osteoporosis Int 2005;16:78-85

2. Barr JD, Barr MS, Lemley TJ, et al. Percutaneous vertebroplasty for pain relief and spinal stabilization. Spine 2000;25:923-28

3. Lieberman I, Dudeney S, Reinhardt MK, et al. Initial outcome and efficacy of 'kyphoplasty' in the treatment of painful osteoporotic vertebral compression fractures. Spine 2001;26:1631-37

4. Phillips FM, Ho E, Campbell-Hupp M, et al. Early radiographic and clinical results of balloon kyphoplasty for the treatment of osteoporotic vertebral compression fractures. Spine 2003;28:2260-67

5. Bouza C, López T, Magro A, et al. Efficacy and safety of balloon kyphoplasty in the treatment of vertebral compression fractures: a systematic review. Eur Spine J 2006;15:1050-67

6. Taylor RS, Taylor RJ, Fritzell P, et al. Balloon kyphoplasty and ver- tebroplasty for vertebral compression fractures: a comparative systematic review of efficacy and safety. Spine 2006;31:2747-55

7. McKiernan F, Faciszewski T, Jensen R, et al. Latent mobility of osteoporotic vertebral compression fractures. J Vasc Intervent Radiol 2006;17:1479-87

8. McKiernan F, Jensen R, Faciszewski T, et al. The dynamic mobility of vertebral compression fractures. J Bone Miner Res 2003;18:24-29

9. Phillips FM, Ho E, Campbell-Hupp M, et al. Early radiographic and clinical results of balloon kyphoplasty for the treatment of osteoporotic vertebral compression fractures. Spine 2003;28:2260-65

10. Wardlaw D, Cummings SR, Van Meirhaeghe J, et al. Efficacy and safety of balloon kyphoplasty compared with non-surgical care for vertebral compression fracture (FREE): a randomised controlled trial. Lancet 2009;373:1016-24

11. Eck JC, Nachtigall D, Humphreys SC, et al. Comparison of vertebroplasty and balloon kyphoplasty for treatment of vertebral compression fractures: a meta-analysis of the literature. Spine J $2008 ; 8: 488-97$

12. Hiwatashi A, Moritani T, Numaguchi $Y$, et al. Increase in vertebral body height after vertebroplasty. AJNR Am J Neuroradiol 2003;24:185-89

13. Teng MM, Wei CJ, Wei LC, et al. Kyphosis correction and height restoration effects of percutaneous vertebroplasty. AJNR Am J Neuroradiol 2003;24:1893-900

14. Hiwatashi A, Westesson PL, Yoshiura T, et al. Kyphoplasty and vertebroplasty produce the same degree of height restoration. AJNR Am J Neuroradiol 2009;30:669-73

15. Mathis JM. Percutaneous vertebroplasty or kyphoplasty: which one do I choose? Skeletal Radiol 2006;35:629-31

16. Voggenreiter G. Balloon kyphoplasty is effective in deformity correction of osteoporotic vertebral compression fractures. Spine 2005;30:2806-12

17. Shindle MK, Gardner MJ, Koob J, et al. Vertebral height restoration in osteoporotic compression fractures: kyphoplasty balloon tamp is superior to postural correction alone. Osteoporos Int 2006;17:1815-19

18. Silva MB, Zaninelli EM, Benato ML, et al. Evaluation of the reduction by ligamentotaxis in thoracolumbar burst fractures. Coluna/ Columna 2010;9:126-31

19. Cawley DT, Sexton P, Murphy T, et al. Optimal patient positioning for ligamentotaxis during balloon kyphoplasty of the thoracolumbar and lumbar spine. J Clin Neurosci 2011;18:834-36

20. Genant HK, Wu CY, van Kuijk C, et al. Vertebral fracture assessment using a semiquantitative technique. J Bone Miner Res 2009;8:1137-48

21. Lee JH, Kwon JT, Kim YB, et al. Segmental deformity correction after balloon kyphoplasty in the osteoporotic vertebral compression fracture. J Korean Neurosurg Soc 2007;42:371-76

22. Baroud G, Crookshank M, Bohner M. High-viscosity cement significantly enhances uniformity of cement filling in vertebroplasty: an experimental model and study on cement leakage. Spine 2006;31:2562-68

23. Lee IJ, Choi AL, Yie MY, et al. CT evaluation of local leakage of bone cement after percutaneous kyphoplasty and vertebroplasty. Acta Radiol 2010;51:649-54

24. Venmans A, Klazen CA, van Rooij WJ, et al. Postprocedural CT for perivertebral cement leakage in percutaneous vertebroplasty is not necessary-results from VERTOS II. Neuroradiology 2011;53:19-22

25. Boonen S, Van Meirhaeghe J, Bastian L, et al. Balloon kyphoplasty for the treatment of acute vertebral compression fractures: 2-year results from a randomized trial. J Bone Miner Res 2011;26:1627-37

26. Rho YJ, Choe WJ, Chun YI. Risk factors predicting the new symptomatic vertebral compression fractures after percutaneous vertebroplasty or kyphoplasty. Eur Spine J 2012;21:905-11 